\title{
Anomalous x-ray scattering study of the growth of inverted quantum hut structures in a Si-Ge superlattice emitting strong photoluminescence
}

\author{
Manjula Sharma, Milan K. Sanyal, ${ }^{*}$ and Biswarup Satpati \\ Saha Institute of Nuclear Physics, 1/AF Bidhannagar, Kolkata 700064, India \\ Oliver H. Seeck \\ Deutsches Elektronen-Synchrotron, DESY, Notkestrasse 85, Hamburg 22607, Germany \\ Samit K. Ray \\ Department of Physics and Meteorology, Indian Institute of Technology Kharagpur, Kharagpur 721302, India
}

(Received 9 January 2014; revised manuscript received 20 March 2014; published 12 May 2014)

\begin{abstract}
The growth process of germanium inverted quantum hut (IQH) structures, which are embedded in a silicon lattice, has been studied using anomalous x-ray scattering techniques. These self-assembled IQH structures exhibit strong photoluminescence (PL) although the number density of the huts is rather small. We show here that these IQH structures form by the intermixing of germanium with previously deposited silicon producing an intriguing composition variation that keeps the out-of-plane lattice parameter of the alloy almost constant. We have identified a zero strain cubic structure, which extends towards the tip of these IQHs to accommodate large-scale interdiffusion of germanium in silicon lattice. A substantial increase in intensity of the PL peak at around $0.8 \mathrm{eV}$ as the temperature is lowered from 70 to $10 \mathrm{~K}$ and a corresponding activation energy of $49 \mathrm{meV}$ indicates that photon induced carriers are predominantly captured at the tip of the embedded quantum hut structures.
\end{abstract}

DOI: 10.1103/PhysRevB.89.205304

PACS number(s): 78.70.Ck, 61.46.-w, 81.15.Hi, 68.35.-p

\section{INTRODUCTION}

Recent progress in the characterization techniques to understand the growth of various germanium $(\mathrm{Ge})$ quantum structures formed in silicon $(\mathrm{Si})$ lattice have made this research field active again as this development may provide us future optical materials for seamless integration with the present Si-based very large scale integration (VLSI) technology [1,2]. Due to $\sim 4 \%$ lattice mismatch of $\mathrm{Si}$ and $\mathrm{Ge}$, such a quantum structure carries an inherent strain that can be controlled to tailor the band gap to the optical communication window. Ge quantum structures embedded in Si-Ge superlattice systems have already exhibited remarkable photoluminescence properties [3-8]. In recent years, developments in electron microscopy and synchrotron x-ray scattering techniques have enabled us to understand the growth mechanism by which these Ge quantum structures form in each Si-Ge interface over the Ge wet layer with the apex pointing towards the top surface and get ordered vertically in a Si-Ge superlattice due to propagation of strain.

Although $\mathrm{Si}$ and Ge can form alloys in any molar ratio, it is well known that $\mathrm{Ge}$ deposited on the $\mathrm{Si}(001)$ surface grows layer by layer for around three monolayers in a typical temperature range of $450-750^{\circ} \mathrm{C}$, which is used for molecularbeam epitaxy (MBE) growth, as barriers to interdiffusion are sufficiently high [9-11]. By scanning tunneling microscopy studies, it has been shown that after one monolayer (ML) of Ge deposition, surface dimerization introduces nonuniform stress fields in the subsurface regions [9,12]. Hence the sites, which are under compressive stress, would prefer Si occupancy while those under tensile stress would favor Ge. Thus, given sufficient kinetic energy, Ge tends to move to the tensile sites

\footnotetext{
*milank.sanyal@saha.ac.in
}

which lie in the third or fourth subsurface layers in order to lower the overall surface energy [9]. In this temperature regime of MBE growth, the Ge interdiffusion into the $\mathrm{Si}$ layer, even with added driving force of stress, is negligible. For two-monolayer coverage of $\mathrm{Ge}$, less than one-fourth of a layer goes in the underlying $\mathrm{Si}(001)$ lattice [4,9-12]. After a critical thickness of three monolayers of Ge, quantum hut structures are formed on top of this Ge wet layer with the tip pointing towards the top surface.

It is expected that as the growth temperature is reduced $\left(\leqslant 500^{\circ} \mathrm{C}\right)$ the interdiffusion of Ge into the underlying Si lattice will become even lower. But on the contrary, it was observed recently by electron microscopy studies that surprisingly large length-scale interdiffusion of $\mathrm{Ge}$ occurs in the underlying Si layers at low growth temperatures $\left(\sim 450^{\circ} \mathrm{C}\right)$, provided the deposited Ge layer thickness is kept between 22 and $38 \AA$ [12-15]. This enigmatic interdiffusion of $\mathrm{Ge}$ in the underlying $\mathrm{Si}$ lattice at lower growth temperature $\left(\leqslant 500^{\circ} \mathrm{C}\right)$ leads to the formation of quantum hut structures within the Si sublayer below the Ge wet layer with the apex pointing down towards the Si substrate. Here, we present results of systematic anomalous $\mathrm{x}$-ray scattering and cross-sectional transmission electron microscopy (XTEM) studies which develop an understanding of the growth process of these inverted quantum hut (IQH) structures embedded in silicon sublayers of a $\mathrm{Si}-\mathrm{Ge}$ superlattice exhibiting rather strong photoluminescence (PL) properties.

The results presented here clearly show that the deposited Ge layer relaxes strain by uniform intermixing with the previously deposited lower $\mathrm{Si}$ layer to form a $\mathrm{Si}_{0.6} \mathrm{Ge}_{0.4}$ wet layer exhibiting an out-of-plane lattice parameter of $5.64 \AA$ and an in-plane lattice parameter close to the silicon value of $5.43 \AA$. The IQH structure forms with its base just below the wet layer. The anomalous $\mathrm{x}$-ray scattering measurements show interesting composition variation of IQH structure from base 
to tip. A simple model calculation showed that the in-plane structure of IQH can be understood as a stack of layers with each layer having a constant in-plane lattice parameter with laterally varying [16] composition. Compositional variation over large length scale was observed near the base of the IQH structure with in-plane and out-of-plane lattice parameters around 5.44 and $5.57 \AA$, respectively. The rim of the IQH structure approaches an alloy composition of $\mathrm{Si}_{0.7} \mathrm{Ge}_{0.3}$ in all the isostructural stacks of layers from base to tip having in-plane lattice parameters varying from 5.44 to $5.52 \AA$. Near the tip region of the IQH structures both in-plane and out-of-plane lattice parameters become $5.52 \AA$. This value of the out-of-plane lattice parameter in the rim and tip region of the IQH is around $1.66 \%$ higher than the $\mathrm{Si}(001)$ lattice parameter and can get accommodated in the surrounding $\mathrm{Si}$ lattice as the critical thickness [4] of such lattice parameter on Si can be around $100 \AA$, which is higher than the Si-layer thickness used here. We also show here that even with a low number density of IQH structures the grown Si-Ge superlattice exhibits a strong PL peak below $70 \mathrm{~K}$ temperature at around $0.8 \mathrm{eV}$ with dominating exciton binding energy of $49 \mathrm{meV}$ that corresponds to a quantum dot of size $75 \AA[17,18]$. This size is consistent with the tip size of the IQH where the photon induced carriers are finally captured for PL emission.

\section{EXPERIMENT}

In anomalous x-ray scattering measurements, two data sets are collected away from and at the x-ray-absorption edge of a particular material to increase the sensitivity of the $\mathrm{x}$-ray scattering technique. In our study we have carried out measurements at the Ge $K$ edge $(11103 \mathrm{eV})$ and away from it $(11043 \mathrm{eV})$ to determine shape, composition, and strain of Ge IQH structures embedded in a Si-Ge superlattice [19-21]. $\mathrm{X}$-ray measurements were performed both at beamline [22] P08 of the synchrotron radiation source PETRA III, DESY, Germany and at the Indian beamline [23] BL-18B, Photon Factory, KEK, Japan. The monochromatic beam in BL-18B at Photon Factory was collimated with a set of beam defining slits having horizontal opening of 1 and $0.1 \mathrm{~mm}$ in the vertical direction and data collection was done by a Cyberstar scintillation detector. For all the diffraction measurements a slit of $1.5 \mathrm{~mm}$ (horizontal) by $0.25 \mathrm{~mm}$ (vertical) was mounted just before the detector to improve the signal to background ratio. At beamline P08 of Petra III, a beam-defining slit setting of dimension 50 by 300 microns was used in the vertical and horizontal direction, respectively, and the data were collected by a position sensitive linear Mythen detector. For grazing incidence diffraction (GID) measurements, the intensity of all the channels was integrated to obtain the data presented here, whereas for XRD measurements a central region of interest (40 pixels) was integrated. During GID measurements the incident angle was kept to be $0.2^{\circ}$, slightly above the critical angle for $\mathrm{Si}$, to allow the X-ray beam to penetrate the $\mathrm{Si}$ cap layer for probing the underlying Si-Ge superlattice. Radial and angular scans were taken around two in-plane diffraction peaks, i.e., around (400) and (800). The XTEM investigations were carried out using a FEI, Tecnai G2 F30, S-Twin microscope operating at $300 \mathrm{kV}$. HAADF-scanning TEM was employed here using the same microscope, which was equipped with a scanning unit and a HAADF detector from Fischione (Model 3000). Cross-sectional TEM specimens were prepared using mechanical thinning down to $20 \mu \mathrm{m}$ followed by $2-\mathrm{kV}$ Ar-ion milling. Low-temperature PL measurements were performed in a closed-cycle helium cryostat, which can be used in the

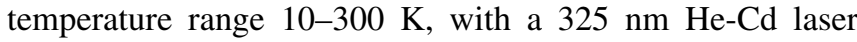
as the excitation source. The power of the laser was kept at $40 \mathrm{~mW}$ during the measurements. The PL spectra were recorded by a liquid-nitrogen-cooled $\mathrm{InGaAs}$ detector using standard lock-in technique.

All the Si-Ge and $\mathrm{Si}-\mathrm{SiGe}$ superlattices presented here were grown on $\mathrm{Si}(001)$ substrates by a solid source MBE system (Compact-21, Riber, France). The base pressure of the growth chamber was $10^{-11}$ Torr. A thick Si buffer layer $(\sim 100 \mathrm{~nm})$ was always grown first before depositing the required superlattice and then a Si cap layer (30-50 nm) was deposited. Figures 1(a)-1(c) show typical TEM micrographs of grown pseudomorphic Si-SiGe alloy superlattice structures by sequential deposition of $\mathrm{Si}$ spacer layers and SiGe alloy layers. These alloy superlattice structures were grown at high temperature $\left(\sim 750^{\circ} \mathrm{C}\right)$ and typical results having an alloy composition of $\mathrm{Si}_{0.43} \mathrm{Ge}_{0.57}, \mathrm{Si}_{0.66} \mathrm{Ge}_{0.34}$, and $\mathrm{Si}_{0.52} \mathrm{Ge}_{0.48}$ are shown in Figs. 1(a)-1(c) respectively. The alloy-layer/Si-layer thicknesses for these superlattice samples were 60/90, 150/260, and 100/175 $\AA$ respectively for the data shown in Figs. 1(a)1(c). The high-resolution TEM images for Figs. 1(a)-1(c) are shown in Figs. 1(A)-1(C) respectively. The high-resolution TEM images clearly show the highly pseudomorphic nature of these superlattices as the SiGe alloy has a lower lattice mismatch with $\mathrm{Si}$ as compared to that of pure Ge. It is also observed that the alloy layers retain the deposited molar ratio and the interfaces remain sharp indicating very little intermixing preferences. In Figs. 1(d)-1(f) we have shown typical superlattice samples of pure $\mathrm{Ge}$ and $\mathrm{Si}$ layers deposited at temperatures of 400,550 , and $500^{\circ} \mathrm{C}$ respectively. The Ge-layer/Silayer thicknesses in these samples were 20/70, 20/110, and 50/300 $\AA$ respectively. Figs. 1(D)-1(F) represent the corresponding high-resolution TEM images for Figs. 1(d)-1(f) respectively. The high contrast observed in these images is due to variation of Ge content in those areas. It is evident from the figures that the strain in Ge layers is relaxed by the formation of IQH structures in (d) and (e), whereas in (f) strain relaxation occurs via introduction of misfit dislocations as the thickness of the Ge layer (=50 $\AA$ ) is far above the critical thickness [4].

\section{RESULTS AND DISCUSSION}

Figure 2(a) shows a typical high-angle annular dark field scanning/transmission electron microscopy (STEM-HAADF) image of a representative superlattice sample and the line across the layers (over the IQH stack) is the profile path for STEM-energy dispersive X-ray (EDX) measurements. The Si and Ge profiles along the line from the Si buffer (top) to the cap layer (bottom) are shown in Fig. 2(b). It can be easily observed that the variation of $\mathrm{Si}$ concentration as a function of depth is exactly opposite to that of the Ge concentration as expected. In Fig. 2(c) we have shown the same Ge EDX profile using bars of equal thickness $(\sim 13 \AA)$ over the lowest three $\mathrm{Si}-\mathrm{Ge}$ bilayers. Since on average four bars are present on 

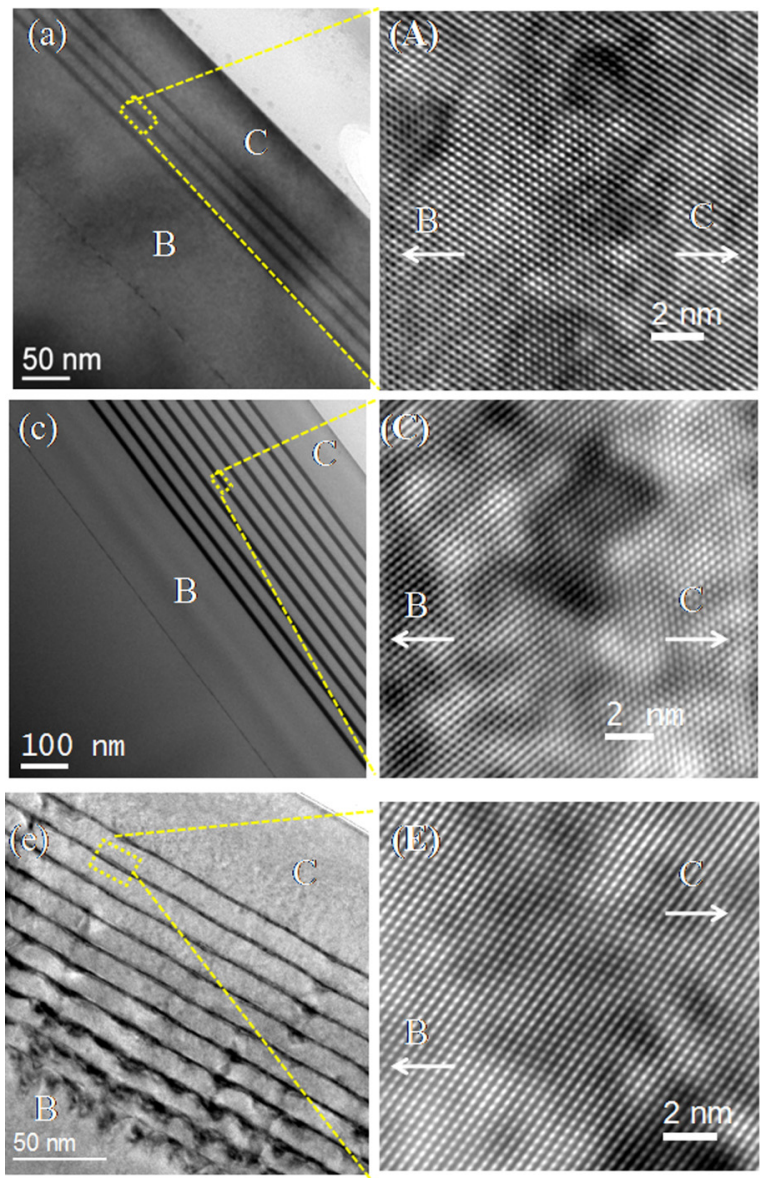
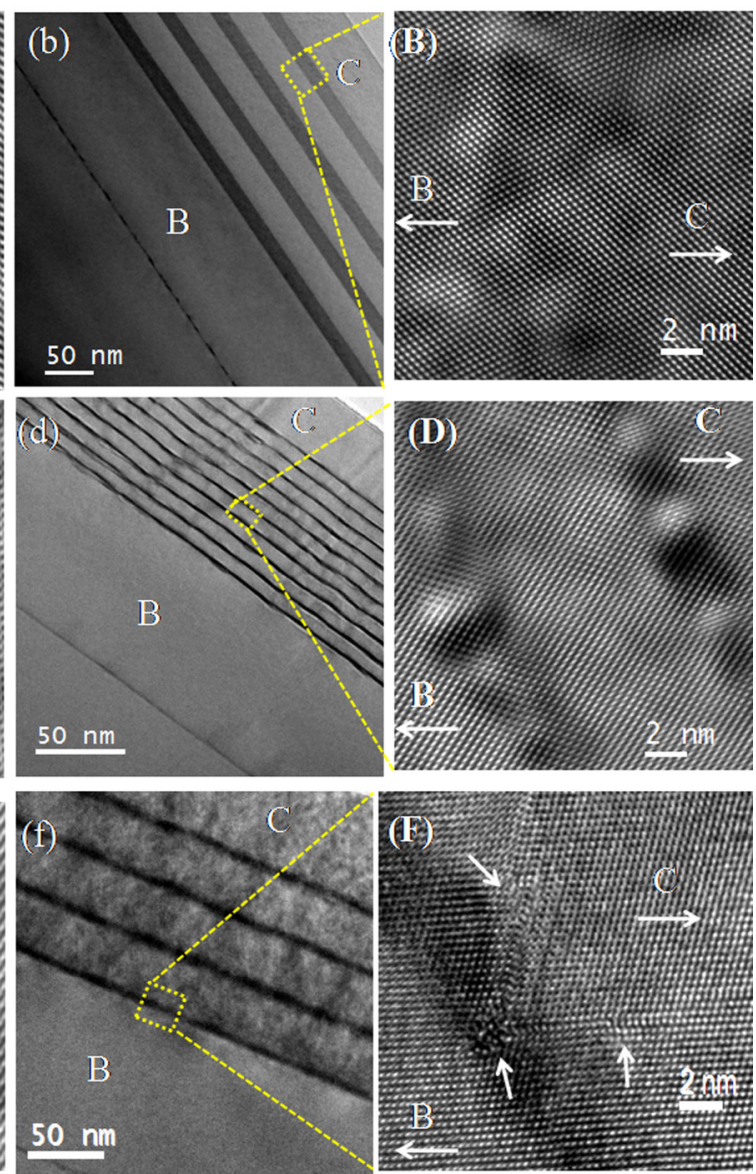

FIG. 1. (Color online) (a)-(c) Si-SiGe alloy superlattices with varied layer thicknesses, grown at $750{ }^{\circ} \mathrm{C}$. These alloy superlattices are seen to be completely pseudomorphic as evident from the high-resolution TEM images (A)-(C) respectively. (d),(e) Si-Ge 10 bilayer superlattices relaxing strain by the formation of IQH stacked one over the other in various layers. (D),(E) Corresponding high-resolution TEM images for (d) and (e) respectively. The high contrast in these images is due to high Ge content in those areas. (f) Si-Ge 4 bilayer sample showing strain relaxation by the introduction of misfit dislocations as indicated in the high-resolution TEM image (F). The misfit dislocations are attributed to large thickness of Ge layer. Buffer (B) and cap (C) layers are indicated in all the images.

the buffer side while only two are on the cap side, obviously the slope of the Ge concentration on both sides of the wet layer are different. The observed small slope of the Ge-over-Si interface is due to the presence of IQH structures. It is to be noted that during the occurrence of all this diffusion, proper epitaxy of the heterostructure is maintained and high-resolution TEM studies have revealed the absence of any plastic relaxation in the system. Hence, even though the Ge diffusion is not random and occurs making a slope with the wet layer to form the observed IQH structure, the theory of elasticity applies in this system.

It is known that the Ge wet layer on a $\mathrm{Si}(001)$ surface has an inherent biaxial compressive strain in the growth plane that leads to tensile strain in the out-of-plane direction [4]. The anomalous GID measurements around the (800) and (400) diffraction peaks provide us direct information regarding strain in the in-plane lattice with respect to the Ge composition profile [19]. The composition and strain information obtained from these GID measurements was used in the analysis [23] of the out-of-plane diffraction data to obtain detailed knowledge about the composition-strain profile of these superlattice systems.

\section{A. In-plane structure}

We have carried out radial and angular scans in the GID measurements. Radial scans are intensity measurement by varying the incidence angle $(\theta)$ to the in-plane lattice and the detector angle $(\Phi)$ position by keeping $\Phi=2 \theta$. The measured $\mathrm{x}$-ray intensity can be directly related to the in-plane lattice parameter $\left(a_{\|}\right)$as $a_{\|}=\lambda \sqrt{h^{2}+k^{2}+l^{2}} /[2 \sin (\Phi / 2)]$, where $(h, k, l)$ are the Miller indices of the nearest Bragg reflection. In angular scans $\theta$ is varied by keeping $\Phi$ (detector position) value constant and the measured $\mathrm{x}$-ray intensity gives the size of the region in the sample having a fixed in-plane lattice parameter corresponding to the fixed $\Phi$ position $\left[q_{r}=(4 \pi / \lambda) \sin (\Phi / 2)\right]$. It is to be noted here that only the angular momentum transfer $\left[q_{a}=(4 \pi / \lambda) \sin (\theta-\Phi / 2)\right]$ changes in these scans while the radial momentum remains constant. Figures $3(a)$ and $3(b)$ show typical GID two-dimensional radial scans of the superlattice sample shown in Fig. 2(a) at two energies around (800). At the Ge $K$ edge, the contribution of the Ge atomic scattering factor in the diffracted $x$ rays is much less [19-21] as compared to this contribution when the $\mathrm{x}$-ray measurement is done away from the Ge $K$ edge. The reduction in Ge scattering at the x-ray edge leads to the reduction in the intensity of the diffraction peak as 

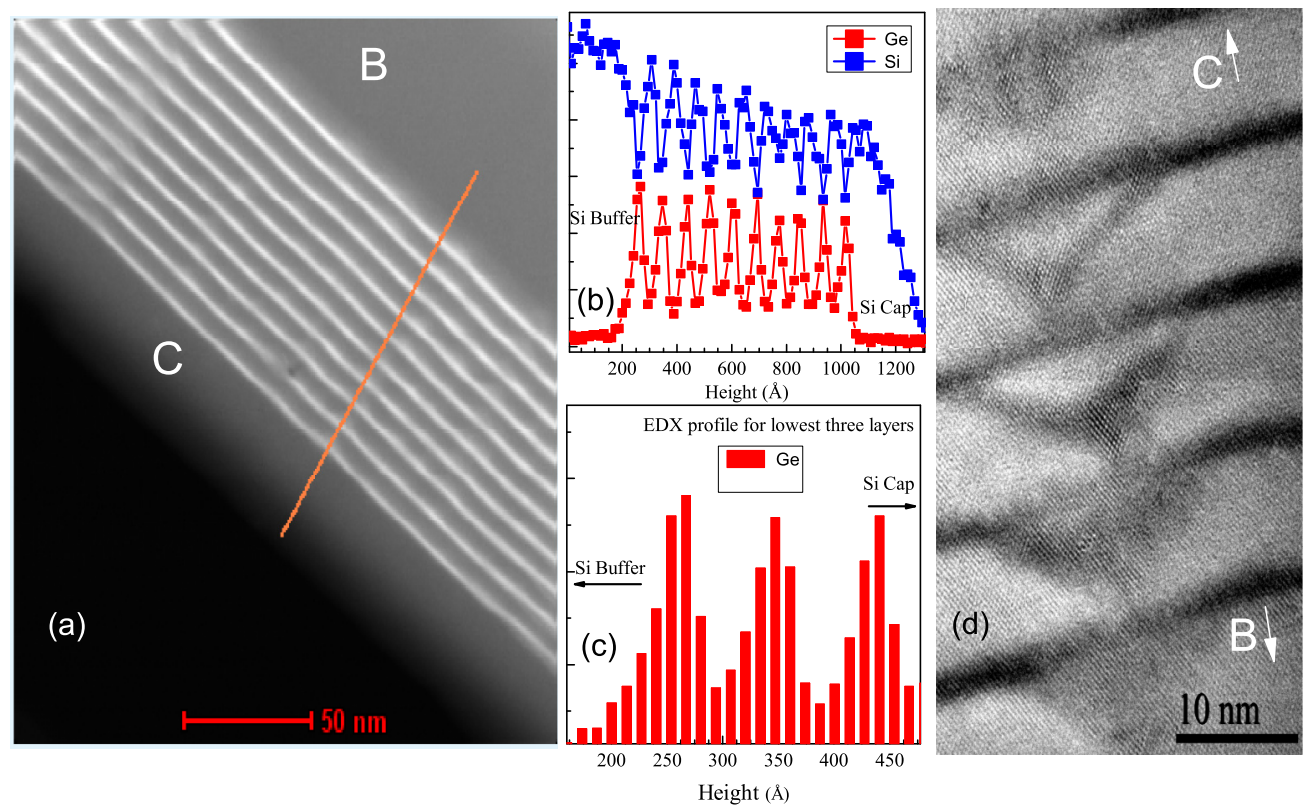

FIG. 2. (Color online) (a) STEM-HAADF image of the superlattice structure. Brighter lines correspond to Ge layers while the gray portions are Si. Buffer (B) and cap (C) layers are indicated. (b) STEM-EDX line profile along the line shown in (a). The line profile is taken over the IQH stack in various layers. (c) Ge EDX profile using bars of equal thickness ( $\sim 13 \AA$ ) for the lowest three Si-Ge bilayers. (d) High-resolution XTEM micrograph of self-organized IQH structures formed in a Si-Ge superlattice sample. B and C indicate the location of buffer and cap layer respectively.

can be seen in radial scans of Fig. 3(c). Hence, by taking the ratio of intensities measured at two energies $E_{1}=11043 \mathrm{eV}$ (away from the Ge $K$ edge) and $E_{2}=11103 \mathrm{eV}$ (at Ge $K$ edge), one can calculate the Ge concentration corresponding to a lattice parameter $\left(a_{\|}\right)$using the formula [19]

$$
x=\left[1+\frac{f_{\mathrm{Ge}_{2}} \sqrt{I_{1}}-f_{\mathrm{Ge}_{1}} \sqrt{I_{2}}}{f_{\mathrm{Si}}\left(\sqrt{I_{2}}-\sqrt{I_{1}}\right)}\right]^{-1},
$$

where $I_{1}$ and $I_{2}$ are the scattered intensities and $f_{\mathrm{Ge}_{1}}$ and $f_{\mathrm{Ge}_{2}}$ are the atomic scattering factors at the energies $E_{1}$ and $E_{2}$ respectively and $f_{\mathrm{Si}}$ is the atomic scattering factor of Si that remains almost the same at the two x-ray energies considered here. We obtained the Ge composition from the analysis of the two radial scans for (800) as a function of the in-plane lattice parameter $\left(a_{\|}\right)$and the result is plotted in Fig. 3(d). To determine the in-plane strain $\left\{\varepsilon_{\|}=\left[a_{\|}-a(x)\right] / a(x)\right\}$, one has to compare the lattice parameter $\left(a_{\|}\right)$with $a(x)$ calculated from the Ge composition obtained from Vegard's law [refer Fig. 3(d)]. In Fig. 3(e) we show the in-plane strain profile thus obtained as a function of $a_{\|}$and from this plot we calculated [refer Fig. 3(f)] the out-of-plane lattice parameter $a_{\perp}$, using Poisson's relation $a_{\perp}=-\frac{2 C_{12}}{C_{11}}\left[a_{\|}-a(x)\right]+a(x)$ where $C_{11}$ and $C_{12}$ are the known components of fourth rank strain tensor related as $\varepsilon_{\perp}=-2 \varepsilon_{\|} C_{12} / C_{11}$ [4]. It is to be noted here that the value of the ratio $C_{12} / C_{11}$ changes from 0.385 to 0.372 as one uses tabulated values of $\mathrm{Si}\left(C_{11}=1.66 \mathrm{Mbar}, C_{12}=\right.$ $0.64 \mathrm{Mbar})$ and $\mathrm{Ge}\left(C_{11}=1.29 \mathrm{Mbar}, C_{12}=0.48 \mathrm{Mbar}\right)$. We have used values of $\mathrm{Ge}$ to extract the out-of-plane lattice parameter $a_{\perp}$ approximately here as the measured data in the out-of-plane direction was not found to be sensitive to this small variation. The sharp feature near the Si peak position (5.43 $\AA$ ) was not used here for the composition and strain analysis as this portion of data is very sensitive to sample alignment and provides information primarily about the Si cap and Si buffer layers [24].

The in-plane and out-of-plane lattice parameters corresponding to the broad peak of Ge concentration shown in regions I and II of Fig. 3(d) represent the Ge wet layer. The peak composition of the wet layer is found to be close to $\mathrm{Si}_{0.6} \mathrm{Ge}_{0.4}$ with in-plane strain of around $-2 \%$ as the $a_{\|}$becomes close to the Si lattice parameter $(5.43 \AA)$ and $a_{\perp}$ approaching the value of $5.62 \AA$. Analysis of the out-of-plane diffraction data, which will be presented next, clearly shows that the thickness of this wet layer with $a_{\perp}=5.62 \AA$ is around $15 \AA$. The interface of regions II and III ( $a_{\|}=5.44 \AA$ ) represent the base of the IQH structure below the wet layer and the second composition peak of $\mathrm{Ge}$ concentration $\mathrm{Si}_{0.7} \mathrm{Ge}_{0.3}$ [refer to the interface of regions III and IV of Fig. 3(d)] represent the tip of IQH. From Figs. 3(c) and 3(d), it can be seen that the Ge concentration becomes small as we approach $a_{\mathrm{Ge}}(=5.65 \AA)$ implying that pure Ge has not precipitated here. It is also clear from Figs. 2(a) and 2(d) that, apart from the wet layer, Ge in alloy form is present only in the IQH structure and from the contrast of the TEM data it is apparent that the Ge concentration is higher in the rim area and tip portion of the IQH structure. It is to be noted here that a similar composition was detected in the rim and in the tip of IQH, in an earlier TEM study [13]. It is also clear from Fig. 3(f) that apart from regions II and I that represent the wet layer having a peak composition of $\mathrm{Si}_{0.6} \mathrm{Ge}_{0.4}$ the out-of-plane lattice parameter $a_{\perp}$ remains almost constant at a value of around $5.52 \AA \pm 0.01 \AA$ (shown in shaded region) though the in-plane lattice parameter $a_{\|}$is changing from 5.35 to $5.52 \AA$. This shaded region can be attributed to the entire IQH structure. From the base of the IQH to its tip, the in-plane lattice parameter $\left(a_{\|}\right)$varies from 5.44 to $5.52 \AA$ 

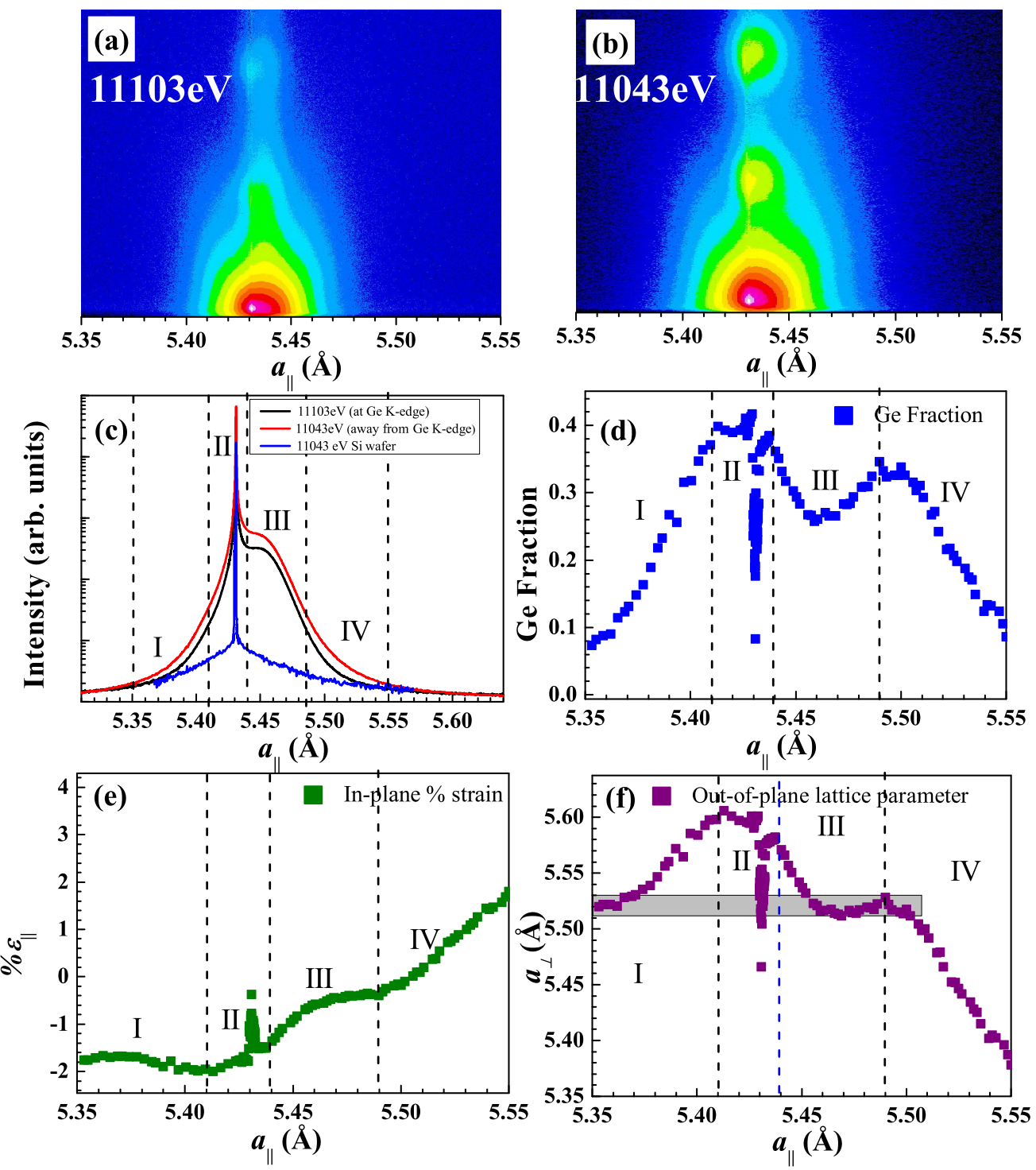

FIG. 3. (Color online) Panels (a) and (b) show two-dimensional GID data of the superlattice sample shown in Fig. 2(a) at the Ge $K$ edge $(11103 \mathrm{eV})$ energy and away from it $(11043 \mathrm{eV})$ respectively around (800). (c) The extracted radial scans for the superlattice structure at the two energies as indicated, around (800). This plot is generated by integrating the counts on Mythen detector for each $a_{\|}$. Also shown is the (800) profile for Si substrate for comparison. (d)-(f) Variation of Ge concentration, in-plane strain, and out-of-plane lattice parameter respectively with the in-plane lattice parameter as obtained from (800) radial scans. Refer to text for details about the shaded region in (f).

keeping the out-of-plane lattice parameter $\left(a_{\perp}\right)$ constant at $5.52 \AA$. At the end of this shaded region, in the tip portion of the IQH, the composition becomes $\mathrm{Si}_{0.7} \mathrm{Ge}_{0.3}$ and the value of the in-plane lattice parameter $a_{\|}$also becomes close to $5.52 \AA$. This structure of the tip region of the IQH has nearly zero strain value [refer the interface of regions III and IV of the in-plane strain profile shown in Fig. 3(e)]. The out-of-plane lattice parameter $\left(a_{\perp}\right)$ of $5.52 \AA$ in the tip and rim structure helps accommodating the IQH in the Si lattice with around $1.66 \%$ strain as compared to the Si lattice. It should be noted that the critical thickness for SiGe heterostructures with $1.66 \%$ strain is around $100 \AA$ [4]. A very small amount of Ge present at the beginning of region I in Fig. 3(d) represents the Si lattice in the vicinity of the IQH structure that matches the out-of-plane lattice parameter $5.52 \AA$ giving rise to $a_{\|}=5.313 \AA$ for $\mathrm{Si}$. The Si lattice near the tip of the IQH structure needs to match the in-plane lattice parameter of $5.52 \AA$ and that in turn requires $a_{\perp}=5.36 \AA$. This lattice is represented towards the end of region IV. However, for simplicity we have not used such an out-of-plane lattice parameter to fit the (004) data presented in the next section. An in-plane lattice parameter lower than that of $\mathrm{Si}\left(a_{\mathrm{Si}}\right)$ was also observed earlier in a system consisting of pits [24]. From the strain profile of Fig. 3(e) it can be seen that with increasing $a_{\|}$, the strain in $\mathrm{Si}_{1-x} \mathrm{Ge}_{x}$ moves from negative to positive values through zero and this represents different portions of the IQH structure. A similar behavior for strain, i.e., changing from compressive to tensile for $a<a_{\mathrm{Si}}$ and $a>a_{\mathrm{Si}}$, respectively, was observed in other SiGe quantum 
dot systems as well [24]. Propagation of such an oscillatory strain profile probably ensures that IQH structures get located on top of each other [refer Fig. 2(d)].

\section{B. Out-of-plane structure}

The out-of-plane diffraction measurements allow us to check consistency of the strain-composition profile obtained from the GID analysis and we show here the results of a typical analysis of data around the silicon (004) diffraction peak. Figure 4(a) shows such XRD data taken at $11043 \mathrm{eV}$ along with the theoretically calculated profile [23], obtained with the Born approximation. For this calculation, we used a profile for $a_{\perp}$ and a corresponding $\mathrm{Si}_{1-x} \mathrm{Ge}_{x}$ composition as obtained from GID measurements [refer to Figs. 3(f) and 3(d)]. Thus, depending upon the composition, each lattice site was assigned with a scattering amplitude which was the weighted average of the scattering amplitudes of $\mathrm{Si}$ and $\mathrm{Ge}$. The fitting of the experimental XRD profile is performed by varying the layer thicknesses and by introducing alloy layers at the interfaces through an iterative process. The final profile of $a_{\perp}$ and the associated strain, defined as $\varepsilon_{\perp}=\left[a_{\perp}-a(x)\right] / a(x)$, and the corresponding $\mathrm{Si}_{1-x} \mathrm{Ge}_{x}$ composition obtained from this fitting of the XRD data are shown in Figs. 4(b)-4(d), respectively. We have also shown an expanded profile of one bilayer in the inset of each figure for clarity. The average out-of-plane lattice parameter, strain, and composition of a typical Ge-Si bilayer are shown in these insets. The beginning of the wet layer and the end of the IQH structure is marked with arrows. The values obtained here are consistent with those obtained from the analysis of the in-plane GID data. The obtained profiles presented in these figures represent the data well. The top $330 \AA$ of the Si cap layer is completely relaxed with lattice parameter $a_{S i}$ as expected from growth conditions. The wet layer of the $\mathrm{SiGe}$ alloy has a thickness of around two to three unit cells $(\sim 15 \AA)$ at all the interfaces and a large diffusion of up to five to six unit cells is seen below the wet layer. The Ge content $(x)$ was found to vary [refer to Fig. 4(d)] from zero for the Si cap and spacer layers to around $(x=0.4)$ for the wet layer and the SiGe alloy composition in the IQH was found to be between $\mathrm{Si}_{0.7} \mathrm{Ge}_{0.3}$ and $\mathrm{Si}_{0.8} \mathrm{Ge}_{0.2}$. Different regions in Ge layers are seen to be under both positive and negative out-of-plane strain [refer Fig. 4(c)] - these values were found to be consistent with the in-plane strain values obtained from the analysis of GID data [refer to Fig. 3(e)].

\section{Growth mechanism}

The analysis of the in-plane and out-of-plane x-ray data clearly shows that IQH structures embedded in the Si lattice have the following three distinct features: (a) the base of the $\mathrm{IQH}$ structure is just below the wet layer that has a $\mathrm{Si}_{0.6} \mathrm{Ge}_{0.4}$ composition with an out-of-plane (in-plane) lattice parameter of around $5.6 \AA(5.4 \AA)$; (b) the IQH structure has a rim and a tip both having a composition of $\mathrm{Si}_{0.7} \mathrm{Ge}_{0.3}$; and (c) the average out-of-plane (in-plane) lattice parameters just below the IQH structure and above the wet layer [marked as arrows in inset of Fig. 4(b)] are $5.43 \AA$ (5.52 $\AA$ ) and $5.46 \AA$ (5.51 $\AA$ ) with low Ge $(<0.2)$ concentration. In the growth model proposed here, we assume that the entire quantum structure of Ge from the wet layer to the IQH tip, which almost touches the next wet layer below, is composed of in-plane isostructural domains
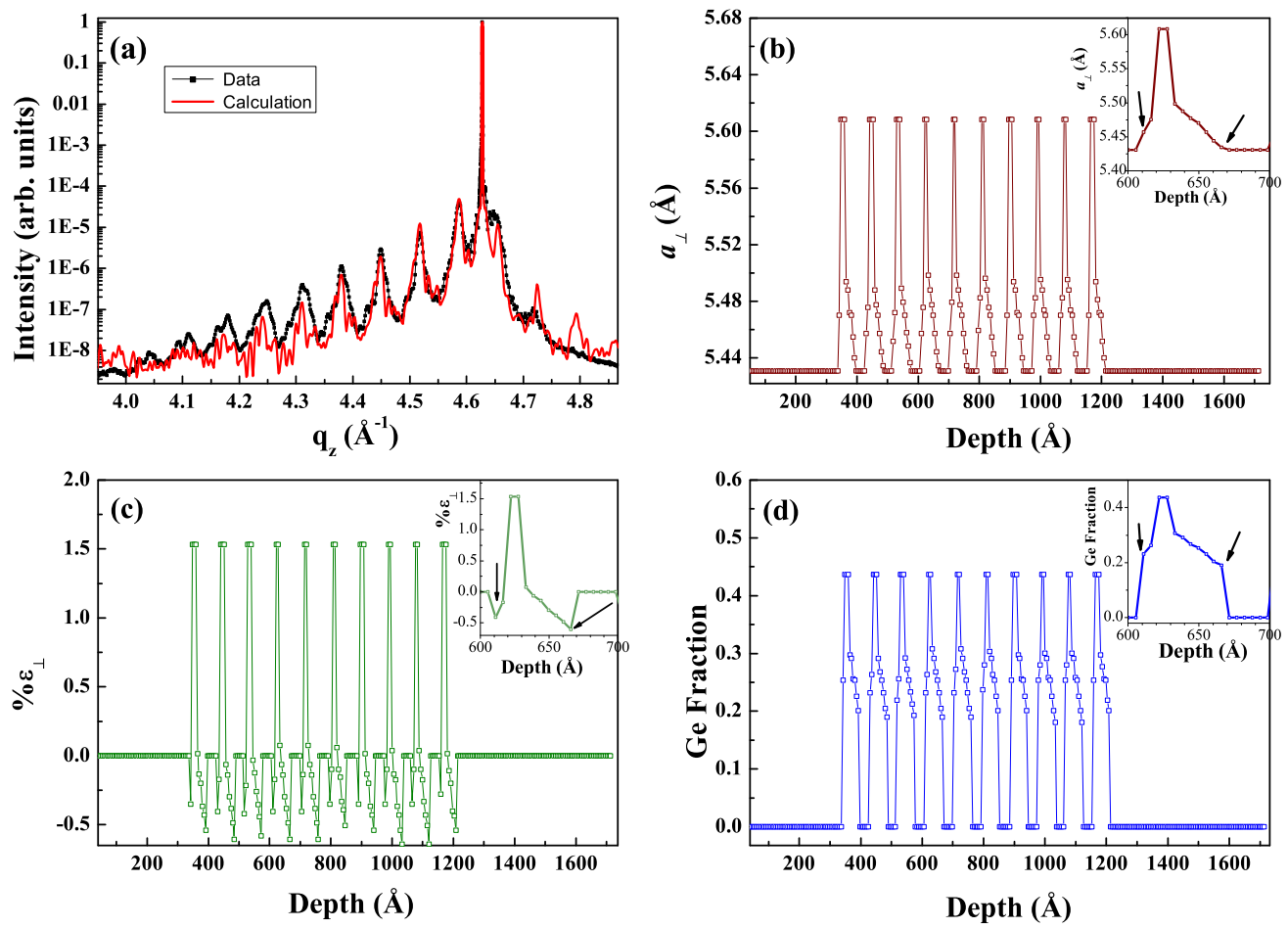

FIG. 4. (Color online) (a) Experimental data and calculated XRD profile of the superlattice structure. (b)-(d) Variation in lattice parameter, out-of-plane strain $\left(\varepsilon_{\perp}\right)$, and Ge fraction $(x)$ respectively with depth as obtained from the XRD data analysis. Insets in (b)-(d) show the expanded version of one bilayer for clarity. 

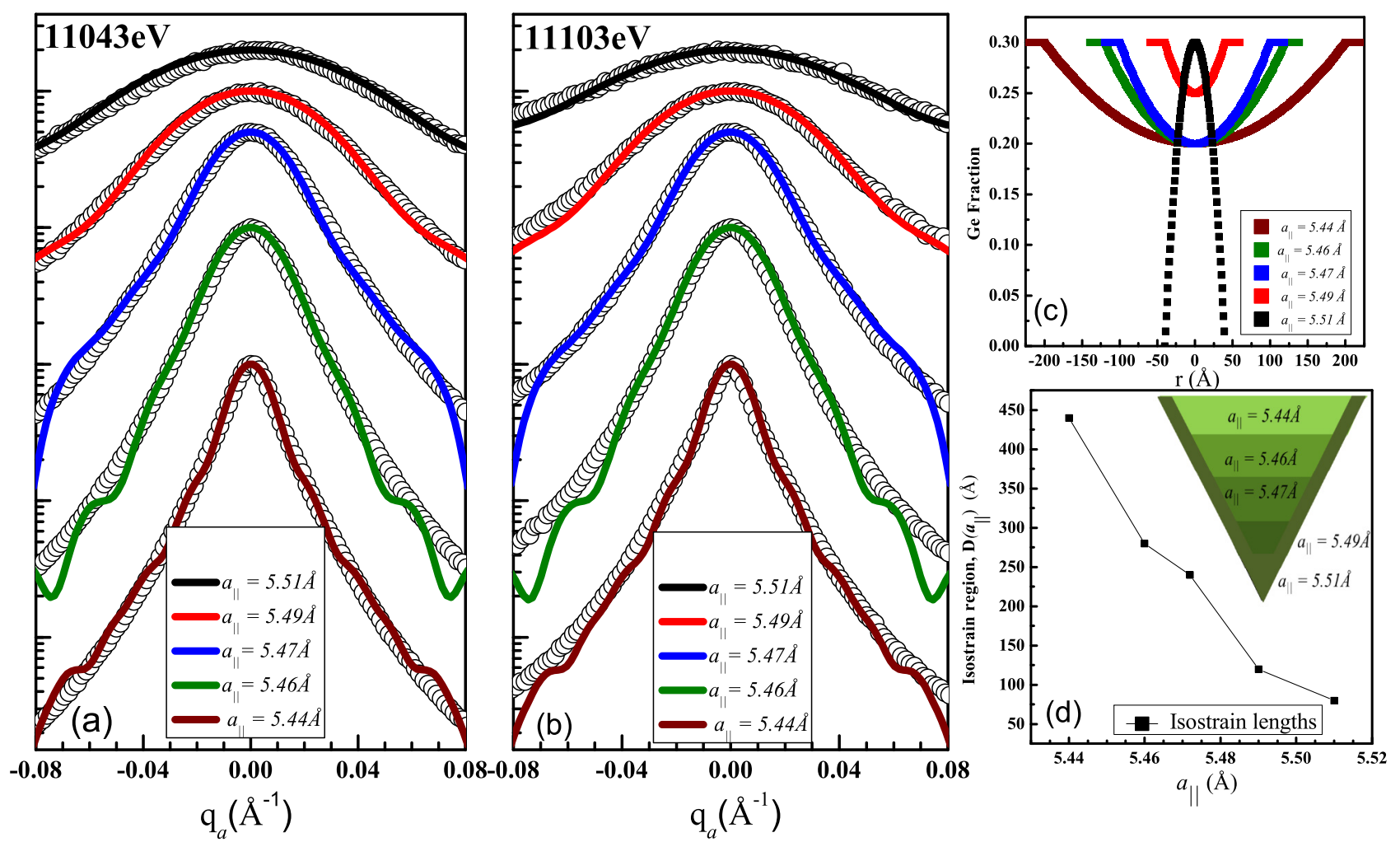

FIG. 5. (Color online) (a),(b) Angular scans around (400) of the superlattice sample measured at $11043 \mathrm{eV}$ and $11103 \mathrm{eV}$ respectively. Each scan is associated with a different $a_{\|}$as indicated in the plot. The solid lines show fits assuming IQH composed of five disks having same in-plane lattice parameter. (c) Ge fraction variation over the disk radially used for fitting. (d) Variation of the isostrain region with $a_{\|}$ as obtained from the fits. Inset shows the schematic (not up to scale) of the IQH being divided into disks of isostrain region. The dark border represents the rim area having higher Ge concentration as observed in the XTEM images and also included in the calculation of fitted curves (refer to text for further details).

with increasing in-plane lattice parameter from regions I to IV [refer Fig. 3(f)]. The domain size of the wet layer was found to be very large $(\sim 6000 \AA)$ from the angular scan and the in-plane lattice parameter of the wet layer ranges from 5.375 to $5.435 \AA$ [regions I and II of Fig. 3(f)].

Figures 5(a) and 5(b) show the typical angular scans taken with 11043 and $11103 \mathrm{eV}$ x-ray beams around the (400) diffraction peak at various fixed radial positions corresponding to different in-plane lattice parameters $a_{\|}$as indicated in each profile. The IQH structure can be considered to be consisting of a stack of disks with diameters, $D\left(a_{\|}\right)(=2 R)$, having equal in-plane lattice parameter $\left(a_{\|}\right)$. Following the contrast of the XTEM image [refer Fig. 2(d)] and an earlier study [16] we assumed here that in each of the disks the composition is not uniform laterally. Hence during calculation a parabolic composition profile was assumed over the disk radially for the inner part and a constant $\mathrm{Ge}$ concentration $\left(\mathrm{Si}_{0.7} \mathrm{Ge}_{0.3}\right)$ of thickness $20 \AA$ was taken for the rim structure. Thus, the x-ray scattering profile can be given as [16]

$$
\begin{aligned}
I\left(q_{a}, R\right)= & \frac{I_{0}}{\pi^{2} R^{4}\left|\left\langle f_{\mathrm{SiGe}}\right\rangle\right|^{2}} \\
& \times\left|\int_{0}^{2 \pi} \int_{0}^{R} e^{-i q_{a} r \cos (\theta)} f_{\mathrm{SiGe}}(r) r d r d \theta\right|^{2},
\end{aligned}
$$

where $f_{\mathrm{Ge}}$ and $f_{\mathrm{Si}}$ are the atomic scattering factors for Ge and $\mathrm{Si}$ respectively. $f_{\mathrm{SiGe}}(r)$ represents the scattering factor of the
SiGe alloy at $r$ and $\left\langle f_{\mathrm{SiGe}}\right\rangle$ is the average scattering factor of the disk. The parabolic variation of the Ge fraction in the inner part is taken as

$$
C_{\mathrm{Ge}}(r)=C_{\mathrm{Ge}}(0)+\left[C_{\mathrm{Ge}}(R)-C_{\mathrm{Ge}}(0)\right] r^{2} / R^{2} .
$$

The composition profiles for the different disks are shown in Fig. 5(c). It is to be noted that only the disk at the tip of the IQH has the maximum $\mathrm{Ge}$ content $\left(\mathrm{Si}_{0.7} \mathrm{Ge}_{0.3}\right)$ at the center and it decays as a parabola towards the edge of the disk. All other four disks have maximum $\mathrm{Ge}$ content $\left(\mathrm{Si}_{0.7} \mathrm{Ge}_{0.3}\right)$ at the rim and minimum at the center. We have used the same model to fit the data collected at the two x-ray energies, at the Ge edge and away from the edge. However, it was apparent from the XTEM images that there are variations of sizes particularly in the base portion of the IQH structure. We used four profiles for the base region $\left(a_{\|}=5.44 \AA\right.$ ), each having the same composition profile but radii and relative strength (indicated in parentheses) with respect to first one are as follows: $220 \AA$ (1), $116 \AA(0.35)$, $75 \AA$ (0.16), and $43 \AA$ (0.06); for $a_{\|}=5.46 \AA$ : $140 \AA$ (1), $87 \AA(0.55)$, and $58 \AA(0.25)$; for $a_{\|}=5.47 \AA$ : $120 \AA$ (1), $80 \AA$ (0.75), and $42 \AA$ (0.3); for $a_{\|}=5.49 \AA$ : $60 \AA$ (1) and $30 \AA$ (0.35); for $a_{\|}=5.51 \AA$ : $40 \AA$. It is to be noted that these profiles and our simple model could fit the data set measured at two energies.

The variation of the size of the isostrain region corresponding to different $a_{\|}$as obtained from the fits of the angular scans 
are shown in Fig. 5(d). It is interesting to note that the isostrain region is largest (around $440 \AA$ ) near the base of the IQH structure below the Ge wet layer as is apparent in the TEM image shown in Fig. 2(d). The size of the isostrain region decreases as $a_{\|}$increases, and from the angular scan in the vicinity of the zero-strain region with $a_{\|}\left(=a_{\perp}=5.52 \AA\right)$, we get a value of around $80 \AA$ [refer to Fig. 5(d)] representing the tip area of IQH. This is consistent with the inverted pyramidal-shaped quantum huts observed in XTEM data with higher Ge contrast at the tip of the IQH [refer Fig. 2(d)].

\section{Photoluminescence}

Low-temperature PL measurements of these IQH structures exhibit the characteristic [25-27] broad peak of Ge quantum dots from 0.71 to $0.9 \mathrm{eV}$ [refer Fig. 6(a)]. Such a peak was not observed in the low-temperature $(10 \mathrm{~K}) \mathrm{PL}$ data of the pseudomorphic $\mathrm{Si} / \mathrm{Si}_{0.52} \mathrm{Ge}_{0.48}$ superlattice structure not having any IQH [refer to Fig. 1(c) for the XTEM image]. Gaussian fitting of the integrated PL intensity $\left(I_{\mathrm{PL}}\right)$ profile clearly shows a continuous reduction in peak position from 0.796 to $0.767 \mathrm{eV}$ as the temperature is raised from 10 to
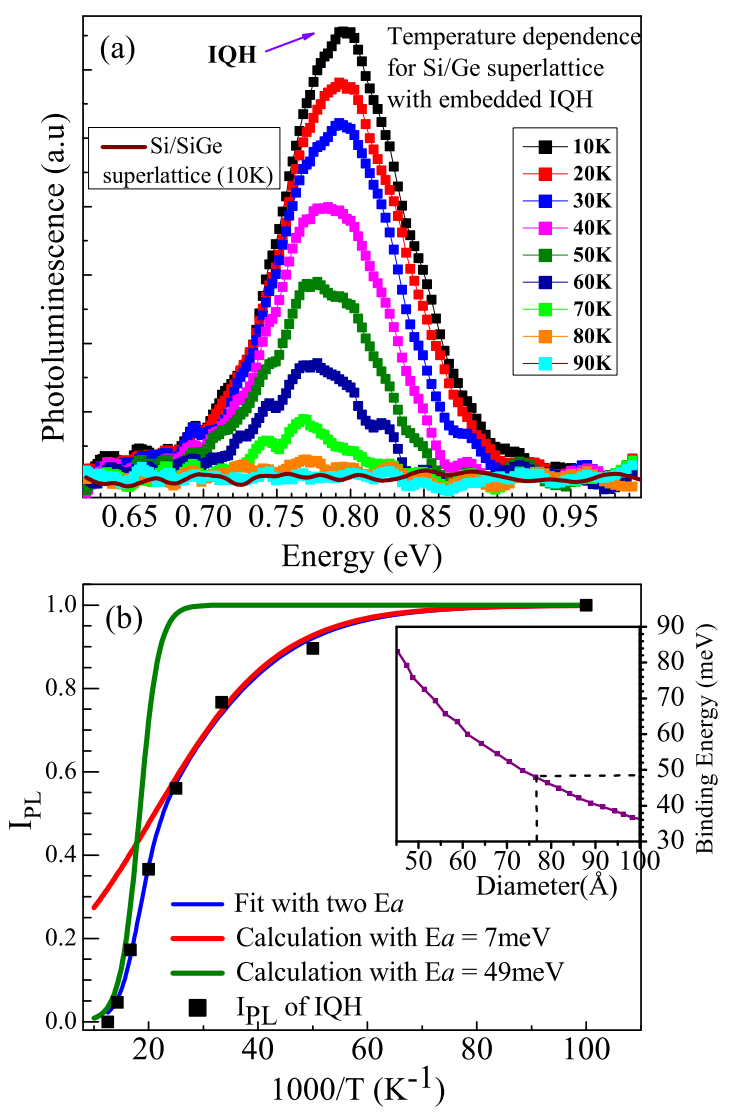

FIG. 6. (Color online) (a) Photoluminescence intensity of the $\mathrm{Si} / \mathrm{Ge}$ superlattice with $\mathrm{Ge} \mathrm{IQH}$ structure at 10 to $90 \mathrm{~K}$ and pseudomorphic quantum well $\mathrm{Si} / \mathrm{SiGe}$ superlattice [Fig. 1(c)] not having quantum hut structures at $10 \mathrm{~K}$. (b) Temperature variation of PL intensity at $0.8 \mathrm{eV}$. The line profile represents the fit considering two activation energies ( 7 and $49 \mathrm{meV}$ ). Also, calculation of the intensity variation is shown for the two activation energies individually which contribute to the final fit.
80 K. A similar shift in PL energy was observed [26] earlier for conventional (noninverted) quantum hut structures. The $I_{\mathrm{PL}}$ was found to decrease as the temperature is increased from 10 to $80 \mathrm{~K}$ [refer Fig. 6(b)]. This variation in intensity can be analyzed using two thermal activation energies [26],

$$
I_{\mathrm{PL}}(T) / I_{0}=1 /\left[1+C_{1} e^{-E_{1} / k T}+C_{2} e^{-E_{2} / k T}\right],
$$

where $I_{0}$ is the maximum PL intensity (at $10 \mathrm{~K}$ ), $C_{1}$ and $C_{2}$ are fitting parameters and $k$ is Boltzmann's constant. The measured data and the fit with Eq. (4) is shown as $I_{\mathrm{PL}}$ vs 1000/T in Fig. 6(b). The obtained two activation energies, $E_{1}$ and $E_{2}$, from the fitting are found to be 7 and $49 \mathrm{meV}$ with corresponding $C_{1}$ and $C_{2}$ values of 5.9 and 55411 respectively. In Fig. 6(b) we have also shown the contribution of the two activation energies. The lower activation energy $E_{1}=7 \mathrm{meV}$ represents the confinement over a large area and is effective only at low temperature. At higher temperature $E_{2}=49 \mathrm{meV}$, representing a quantum confinement over a very small area, dominates. In the inset of Fig. 6(b), we have shown that this activation energy corresponds to $75-\AA$ diameter quantum dots and this is the tip of the IQH structure. The other activation energy of $7 \mathrm{meV}$ may represent the base area of IQH. We could not detect in this measurement PL peaks corresponding to the wet layer and to the transverse-optical (TO) phonon-assisted transitions of $\mathrm{Si}$. This observation indicates that photon induced excitons are primarily captured by the IQH structure and recombination to emit PL photons occurs predominantly at the tip of IQH structures.

\section{CONCLUSIONS}

In conclusion, we have shown here that the IQH structure forms in the Si lattice through enigmatic large-scale diffusion of Ge through formation of $\mathrm{Si}_{1-x} \mathrm{Ge}_{x}$ alloys that minimize the variation of the out-of-plane lattice parameter. We have presented a simple model to explain the growth mechanism of IQH. In this model the IQH structure is composed of a few stratified layers having a constant in-plane lattice parameter with varying composition to represent the rim of the IQH. As we approach the tip of the IQH from the base, the in-plane lattice parameter approaches the out-of-plane lattice parameter to produce a zero-strain cubic lattice having $\mathrm{Si}_{0.7} \mathrm{Ge}_{0.3}$ composition. The temperature-dependent PL study shows that the photon induced carriers are predominantly captured at the tip of the IQH structure. It is important to note that the 25 times increase in the intensity of the PL peak was observed here as the temperature is lowered from 70 to $10 \mathrm{~K}$. It should be possible to enhance the photoluminescence properties of these $\mathrm{SiGe}$ superlattices with inverse quantum hut structures by increasing the number density of IQH structures with appropriate seeding.

\section{ACKNOWLEDGMENTS}

The authors would like to acknowledge the support of Department of Science and Technology (DST) for carrying out synchrotron experiments at Photon Factory, KEK, Japan and at Petra III, DESY, Germany through the DST-KEK and DST-DESY projects operated through Saha Institute of Nuclear Physics. 
[1] A. Morello, Nat. Nanotechnol. 8, 233 (2013).

[2] N. Hrauda, J. Zhang, E. Wintersberger, T. Etzelstorfer, B. Mandl, and J. Stangl, Nano Lett. 11, 2875 (2011).

[3] D. Grutzmacher, T. Fromherz, C. Dais, J. Stangl, E. Muller, Y. Ekinci, H. H. Solak, H. Sigg, R. T. Lechner, E. Wintersberger, S. Birner, V. Holy, and G. Bauer, Nano Lett. 7, 3150 (2007).

[4] K. Brunner, Rep. Prog. Phys. 65, 27 (2002).

[5] D. J. Paul, Adv. Mater. 11, 191 (1999).

[6] H. C. Chen, C. W. Wang, S. W. Lee, and L. J. Chen, Adv. Mater. 18, 367 (2006).

[7] K. L. Wang, D. Cha, J. Liu, and C. Chen, Proc. IEEE 95, 1866 (2007).

[8] P. Boucand, S. Sauvage, M. Elkurdi, E. Mercier, T. Brunhes, V. Le Thanh, D. Bouchier, O. Kermarrec, Y. Campidelli, and D. Bensahel, Phys. Rev. B 64, 155310 (2001).

[9] F. Liu and M. G. Lagally, Phys. Rev. Lett. 76, 3156 (1996).

[10] R. M. Tromp, Phys. Rev. B 47, 7125 (1993).

[11] J. Tersoff, Phys. Rev. B 43, 9377 (1991).

[12] F. Liu, F. Wu, and M. G. Lagally, Chem. Rev. 97, 1045 (1997).

[13] H. H. Cheng, W. P. Huang, V. I. Mashanov, and G. Sun, J. Appl. Phys. 108, 044314 (2010).

[14] H. H. Cheng, C. T. Chia, V. A. Markov, X. J. Guo, C. C. Chen, Y. H. Peng, and C. H. Kuan, Thin Solid Films 369, 182 (2000).

[15] Y. L. Soo, G. Kioseoglou, S. Huang, S. Kim, Y. H. Kao, Y. H. Peng, and H. H. Cheng, Appl. Phys. Lett. 78, 3684 (2001).

[16] A. Malachias, S. Kycia, G. Medeiros-Ribeiro, R. MagalhãesPaniago, T. I. Kamins, and R. S. Williams, Phys. Rev. Lett. 91, 176101 (2003).
[17] T. Takagahara and K. Takeda, Phys. Rev. B 46, 15578 (1992).

[18] C. S. Peng, Q. Huang, W. Q. Cheng, J. M. Zhou, Y. H. Zhang, T. T. Sheng, and C. H. Tung, Phys. Rev. B 57, 8805 (1998).

[19] T. U. Schulli, J. Stangl, Z. Zhong, R. T. Lechner, M. Sztucki, T. H. Metzger, and G. Bauer, Phys. Rev. Lett. 90, 066105 (2003).

[20] M. K. Sanyal, S. K. Sinha, A. Gibaud, K. G. Huang, B. L. Carvalho, M. Rafailovich, J. Sokolov, X. Zhao, and W. Zhao, Europhys. Lett. 21, 691 (1993).

[21] R. Magalhaes-Paniago, G. Medeiros-Ribeiro, A. Malachias, S. Kycia, T. I. Kamins, and R. S. Williams, Phys. Rev. B 66, 245312 (2002).

[22] O. H. Seeck, C. Deiter, K. Plaum, F. Bertam, A. Beerlink, H. Franz, J. Horboch, H. Schulte-Schrepping, B. M. Murphy, M. Greve, and O. Magnussen, J. Synchrotron Radiat. 19, 30 (2012).

[23] M. Sharma, M. K. Sanyal, M. K. Mukhopadhyay, M. K. Bera, B. Saha, and P. Chakraborty, J. Appl. Phys. 110, 102204 (2011).

[24] M. S. Leite, J. L. Gray, R. Hull, J. A. Floro, R. MagalhãesPaniago, and G. Medeiros-Ribeiro, Phys. Rev. B 73, 121308(R) (2006).

[25] M. Larsson, A. Elfving, W. X. Ni, G. V. Hansson, and P. O. Holtz, Phys. Rev. B 73, 195319 (2006).

[26] H. Yang, Z. Tao, J. Lin, F. Lu, Z. Jiang, and Z. Zhong, Appl. Phys. Lett. 92, 111907 (2008).

[27] C. G. Van de Walle and R. M. Martin, Phys. Rev. B 34, 5621 (1986). 